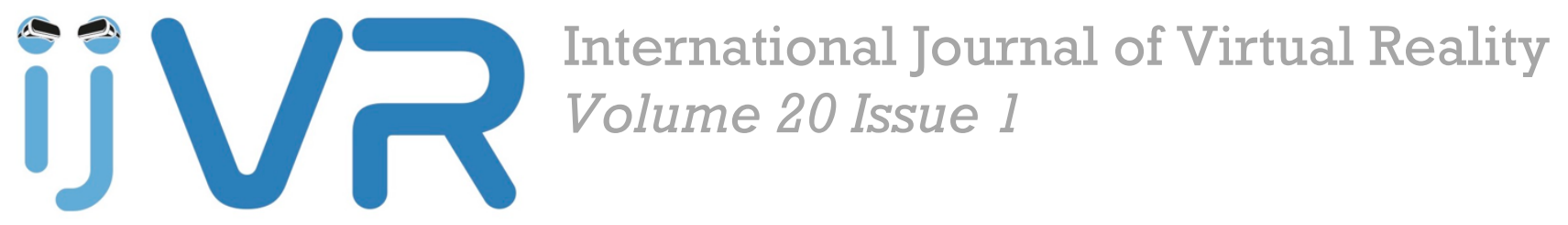

Delgrange R., Burkhardt J.-M., \& Gyselinck V. (2020). Exploring human behavior with Grand Theft Auto $V$ : A study of assisted cognition in wayfinding. International Journal of Virtual Reality, 20(1), 33-47. https://doi.org/10.20870/IJVR.2020.20.1.3249

CC BY 4.0 license

Received: $1 / 2020$

Published: 4/2020 


\title{
Exploring human behavior with Grand Theft Auto V: A study of assisted cognition in wayfinding
}

\author{
Romain Delgrange ${ }^{1,2}$, Jean-Marie Burkhardt ${ }^{1,2}$, Valérie Gyselinck ${ }^{1,2}$ \\ ${ }^{1}$ LAPEA, Univ. Gustave Eiffel, IFSTTAR, Versailles, France \\ ${ }^{2}$ Université de Paris, LAPEA, Boulogne-Billancourt, France
}

Corresponding author: Romain Delgrange, r.delgrange@gmail.com

Keywords: virtual reality - video games - user experience - spatial navigation - navigational aid

\begin{abstract}
The rise of virtual reality has overcome many of the methodological challenges faced by researchers studying spatial navigation. Yet, the difficulty in developing life-like virtual settings still remains a substantial barrier to most studies when fidelity to the real world has to be achieved in order to ensure some psychological validity of the results. Moreover, the variety of the settings results in standardization issues across studies. Our argument is that widely available video games could represent a worthwhile alternative to laboratory-made virtual environments, while providing a satisfactory methodological quality. This study assessed a prototype of landmark-based navigational aid by administering wayfinding tasks in the video game Grand Theft Auto V. Our results provide evidence that this video game offers a transparent and adaptable way to investigate cognitive processes with high experimental control and psychological validity. Recommendations towards the use of video game-based methodologies for future research are discussed.
\end{abstract}

\section{Introduction}

\subsection{Spatial navigation and wayfinding}

Spatial navigation within an environment consists in starting from a location to reach a destination either indoors or outdoors. This is the fundamental first step in most people's daily activities. This activity is sustained by two key components: Locomotion and "wayfinding" (Montello, 2005). While locomotion designates bodily movements and muscular coordination in the immediate surroundings of an individual, moving towards a distant location involves the set of cognitive processes referred to as "wayfinding", defined as the construct and the use of internal spatial representations to find and follow a path linking two places (Golledge, 1992; Montello, 2005, 2017; Wiener et al., 2009). This spatial problem-solving involves not only visual perception, but also executive and mnesic functions, in order to retain spatial information and perform the adequate actions that will lead an individual from an origin to their desired destination (Meneghetti et al., 2017; Vandenberg, 2016). In particular, wayfinding relies heavily on internal spatial representations, or "mental models", from a "landmark", first-person perspective to a "survey", bird's eyeview of the environment (Johnson-Laird, 1980; Siegel \& White, 1975; Tolman, 1948).

Wayfinding is considered a complex, multifactorial and cognitively costly task. Various navigational aids have been developed over the last 40 years in order to assist people in finding their way towards a destination, whether they drive a vehicle or walk (Grison \& Gyselinck, 2019). However, as Grison and Gyselinck (2019) highlight, few of these solutions, if any, rely on the body of work of cognitive science on wayfinding. Indeed, while it is documented that the use of "landmark" spatial information eases wayfinding (Denis, 1997; Denis et al., 2007, 2014), most current navigational aids rely solely on complex "survey” aerial information, and might therefore not be optimal (Grison \& Gyselinck, 2019). Denis et al. (2007) advocated the use of landmark representations in navigational aids, as they have proven crucial to effective wayfinding, allowing people to avoid mistakes and hesitations. However, very few studies have reported testing navigational aids using landmark representations. 


\subsection{Virtual reality as a means to explore human wayfinding in simultaneously controlled and realistic environments}

The study of human behavior in the real world has long faced a methodological limitation, mainly due to the heterogeneous place-specific complexity of a real environment, and the lack of control on it (Kimura et al., 2017). In order to achieve ecological and controllable experimental conditions, researchers faced the need to make the laboratory "life-like" (Gibson, 1979). This methodological challenge has been increasingly overcome in the last 20 years with the rise of virtual reality (Bohil et al., 2011; Tarr \& Warren, 2002). In particular, virtual environments represent the preferred choice for exploring human spatial cognition (Wilson, 2013), as they can potentially reach a high level of fidelity to the physical, perceptual and spatial characteristics of the real world (Burkhardt, 2003). Virtual reality has thus been used in a variety of recent studies on spatial navigation (Gyselinck et al., 2013; Lhuillier et al., 2018; Nys et al., 2018; Plancher et al., 2018).

Yet, since no turnkey solution is available for virtual environments, when using virtual reality, researchers need to develop laboratory-made virtual environments from scratch (Khan \& Rahman, 2018; Li et al., 2019; Lingwood et al., 2018; Pazzaglia et al., 2017; Sharma et al., 2017). Unfortunately, the technical burden of developing functional and life-like virtual settings often restricts the degree of fidelity of the environment to the real world that is achieved. This limitation in fidelity might therefore be a barrier to the "psychological fidelity" of the virtual environment, i.e. the way virtual reality triggers life-like behavior (Leplat, 1997). Thus, as environments achieving heterogeneous levels of fidelity to the real world could indeed result in different behavioral measurements, laboratory-made virtual environments might represent a limitation to the generalizability of the results as well as the replicability of the studies using them.

To our mind, widely available video games could overcome these limitations, as they can provide a high degree of fidelity to the real world and can be used across laboratories as well as being controllable, low-cost and safe experimental tools. Still, video games are rarely, if ever, used as such in the literature (Coutrot et al., 2019). Research on cognitive science and spatial navigation could nevertheless benefit from video game-based methodologies.

\subsection{Objectives of the study}

The present study has two main objectives. Firstly, we aim to assess the effect of two types of navigational aids on human wayfinding performance. More specifically, we compared an innovative landmark-based prototype of navigational aid to a more commonly-used, survey-based wayfinding aid. We also compared the perceived usability of these two aids.

Our second objective was to gather evidence on the use of a widely available video game as a transparent, easily replicable and adaptable means of investigating cognitive processes with both a high experimental control associated to high ecological validity. We used the video game Grand Theft Auto $V$ (Rockstar Games, 2013) to develop the experimental environment and spatial tasks considered in the study.

The present paper reports on part of a larger study, which investigated the effect of cognitive load and navigational assistants on wayfinding and internal spatial representations. In this paper, we focus only on the effect of navigational assistants on wayfinding and usability, and wish to demonstrate the approach used and the interest of using this type of videogame for research on spatial cognition topics.

\subsection{Hypotheses}

We conducted this study with two hypotheses. First, a navigational aid based on landmark information would result in better wayfinding performance during an urban journey, in comparison to a navigational aid based on survey information. Second, we also hypothesized that the landmark-based aid would be evaluated as more usable than the survey-based aid. 


\section{Material and methods}

\subsection{Participant recruitment and sample characteristics}

Forty-six participants (29 women, 17 men) were recruited among the students at the Université de Paris (France). An exclusion criterion was the existence of disabilities impacting the visual, motor or cognitive functions, thereby creating difficulties in wayfinding possibly unrelated to the experimental settings. Participants were informed that they would receive course credits for their participation. The mean age was 21.87 years $(\operatorname{Min}=16, \operatorname{Max}=44, \mathrm{SD}=3.09)$.

\subsection{Experimental conditions}

Participants were randomly assigned to one of the two experimental "Navigational assistant" conditions $(n=22, n=24)$, each comprising a similar proportion of men and women. Each participant travelled a total of two routes, in two different neighborhoods of the virtual environment, for an approximate duration of 20 minutes. The order of the districts was counterbalanced.

The two experimental conditions were based on the navigational assistant assigned to the participant ("landmark" or "survey"). The two assistants gave different instructions for the same route, according to the following principles.

Both navigational assistants gave directions divided into three successive steps (initial, intermediate and closing statements). Both assistants started and closed the guidance with the same initial and closing statements (“You are near the theater...”; “...Then stop at the intersection”). However, the information provided at each intermediate statement during the route differed between the two conditions.

The "landmark" assistant's intermediate statement associated the previously mentioned landmark with an action to be performed, from the point of view of the participant ("Go along this theater on the right..."). The "survey" assistant's intermediate statement gave a bird's eye-view direction that was independent of the participant's point of view ("Head southwest..."). The operation of the two navigational assistants is illustrated in Figure 1.

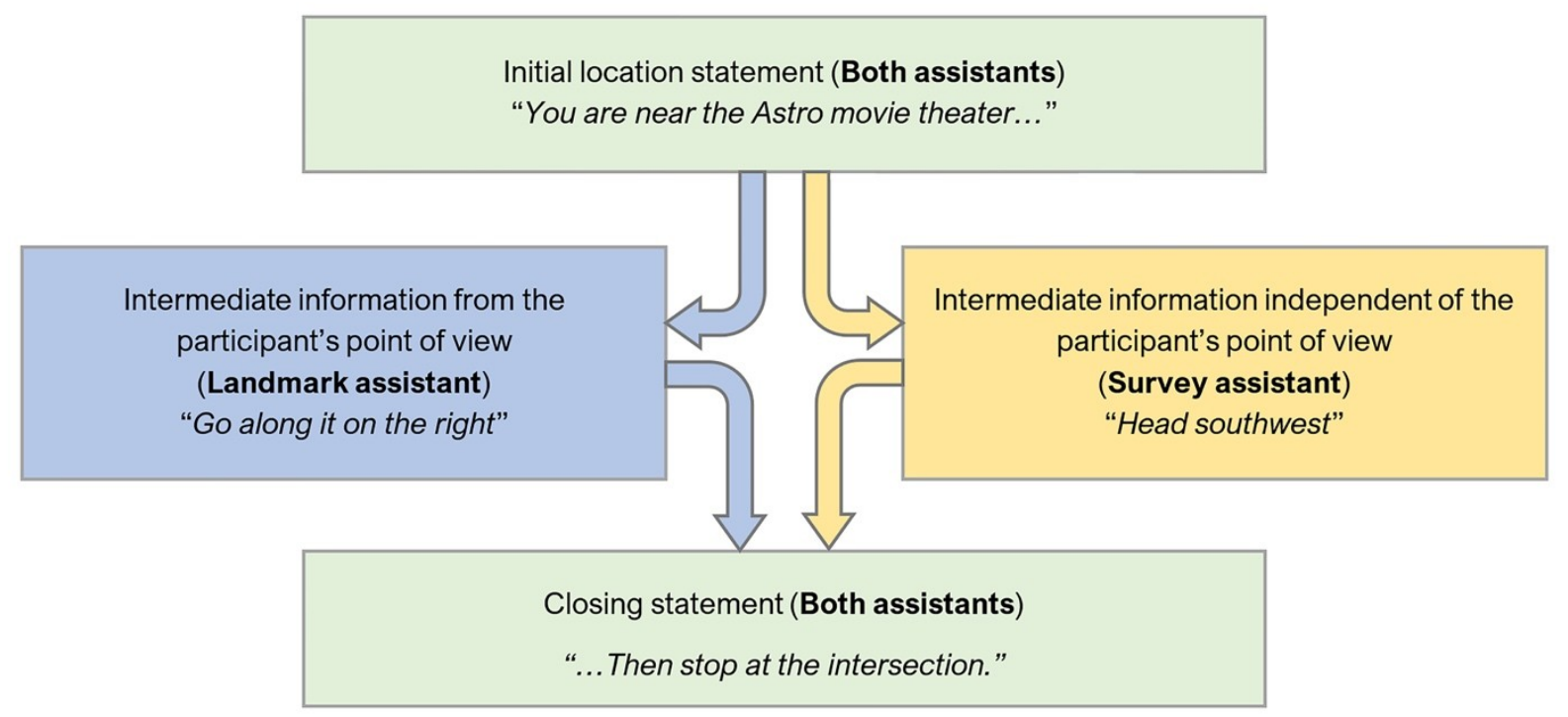

Figure 1: Representation of the operation of the two navigational assistants used in the experiment.

The initial and closing statements of the instruction are always the same regardless of the assistant. Only the intermediate statement guiding the participant differs between an instruction using landmark-based information (landmark assistant) or bird's eye-view information independent of the participant's point of view (survey assistant).

Wayfinding performance was measured from the navigation task in the virtual environment using indicators of effectiveness and efficiency of wayfinding. We measured the moves in the wrong direction during the trip to evaluate wayfinding effectiveness, and visual explorations and complete stops to evaluate 
wayfinding efficiency. Subjective usability was assessed through a dedicated questionnaire, the System Usability Scale (Brooke, 1996).

Spatial skills and prior knowledge of the video game were assessed for both groups in order to ensure that they were equivalent.

\subsection{Wayfinding task}

All participants, regardless of their assigned navigational assistant, performed the wayfinding task. The task consisted in the completion of a total of two routes, in two different virtual neighborhoods. The experimenter initially told the participant that throughout the experiment, the time of day in the virtual environment would be early morning and the sun would therefore always be in the east. The experimenter gave the following instruction to the participant for each neighborhood: "You are sightseeing in a city you do not know. You are looking to visit two shops. You do not know how to get there, but you have a navigational assistant that will guide you along the way". The experimenter then put a reminder in the form of a paper card under the participant's screen where the names of the two shops to be reached during the trip were written, so that the names of their destinations were always visible.

Each of the two trips therefore took the participant from their starting point to two successive destinations: A first and then a second shop. The routes connecting the starting point and the two shops in each neighborhood passed through several decision points, where the navigational assistant had to be used. Each trip was completed when the participant reached the second shop. A representation of the routes in each neighborhood is shown in Figure 2.

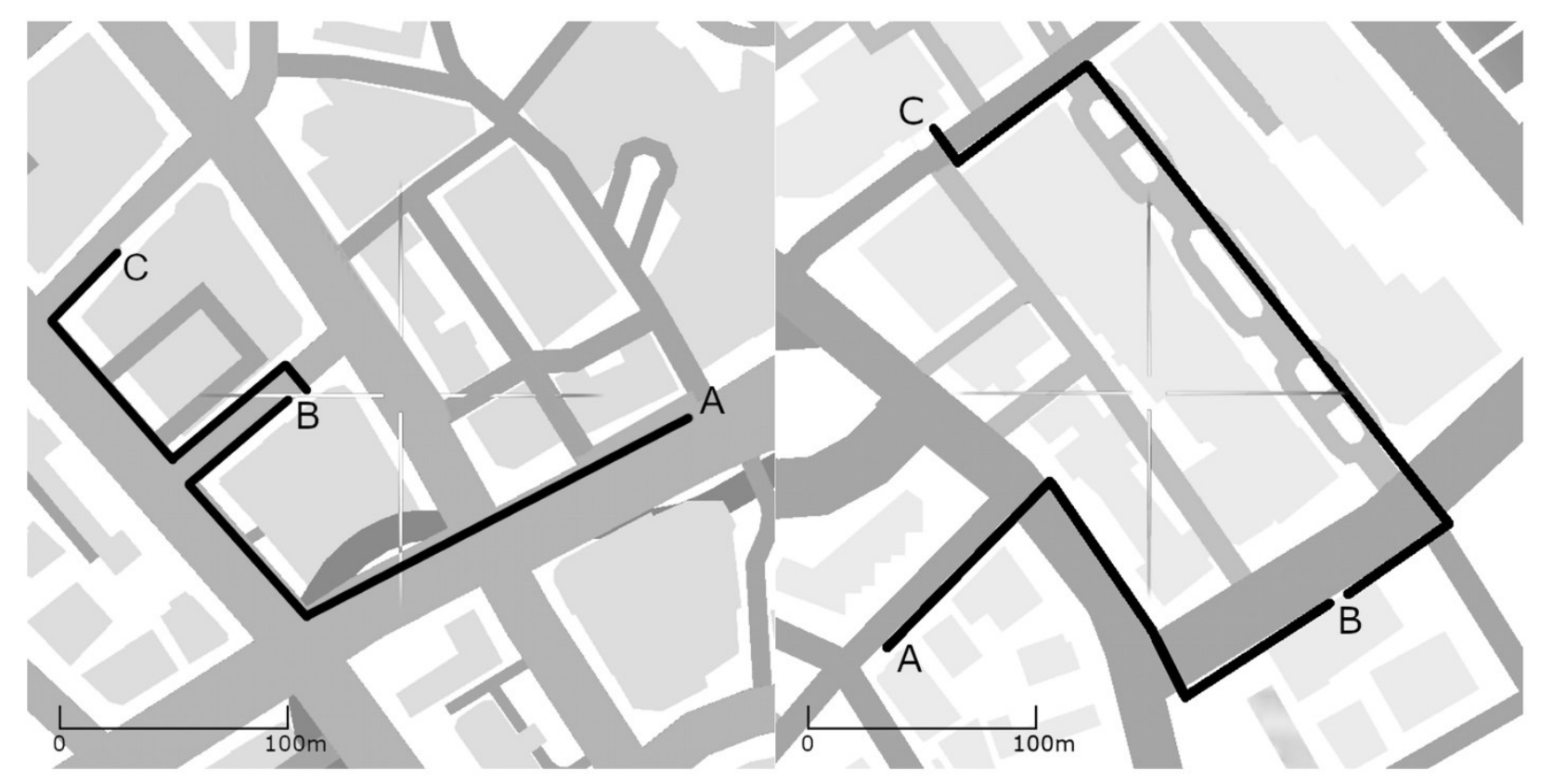

Figure 2: Map representations of the routes in the two virtual neighborhoods.

On each map, (A) indicates the participant's starting point, (B) the first shop to be reached and (C) the second shop. The participant is guided by the navigational assistant during the journey represented by the black line connecting successively (A), (B) and (C). These routes are invariable for all participants.

The participant's starting point was located in front of a single-storey restaurant whose name was displayed on the front. The trip in the first neighborhood passed through seven decision points where the participant had to follow the directions given by the navigational assistant, and led successively to a jewelry store and a clothing store. The trip in the second neighborhood passed through five decision points, and led successively to a fast-food restaurant and an organic shop. All four destination stores were identifiable with a sign indicating their name above their entrance. An example of a destination is pictured in Figure 3. 


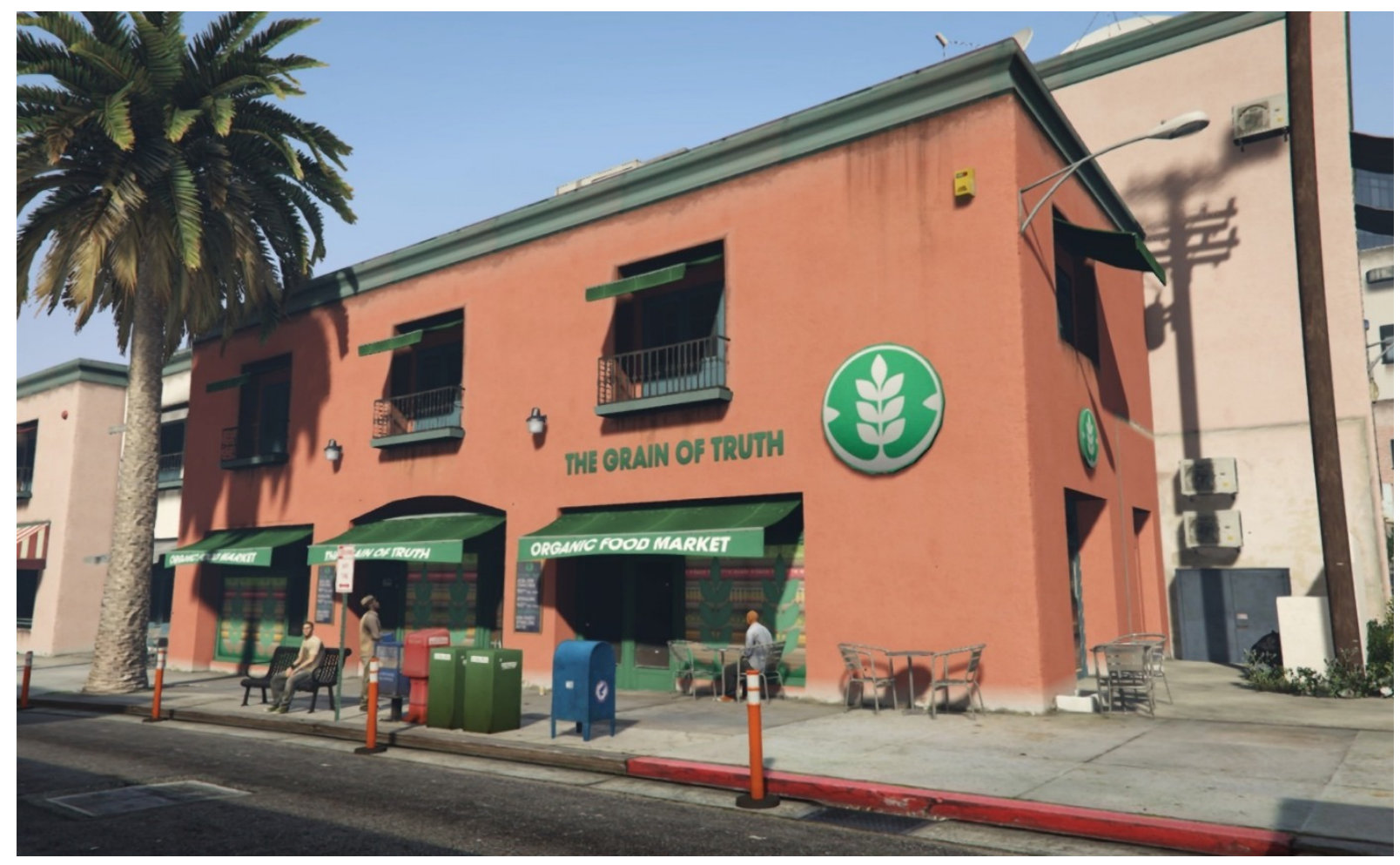

Figure 3: Example of destination for one of the trips, as seen in-game from the street.

The name of each destination was always displayed above the entrance.

The participant had to move towards their destinations thanks to the navigational assistant's instructions, which led them from one decision point to the next. The first instruction of each of the two routes was given automatically by the navigational assistant at the start of each trip. Then, the participant could use the assistant freely during the rest of the trip, by issuing two voice commands, "repeat" and "next", in order to hear the last instruction again or to obtain the next instruction when they had arrived at a decision point and needed to know where to go. The participant had to utter one or the other of the two words distinctly when necessary or when desired to trigger the appropriate instruction.

\subsection{Procedure}

The procedure was in three steps: a training phase ( 5 minutes) followed by two guided trips in the virtual district (10 minutes each). During the training stage, the participant was guided by the experimenter to become familiar with the tasks and the interactions required to easily navigate in the virtual environment (walking and head movements in all directions). Then the navigational assistant was presented to the participant, who had to find the route to two nearby shops, using the assistant. When the participant judged that they could move easily, the training was stopped. Then the wayfinding task was performed. After finishing the task, the questionnaire assessing the usability of the navigational assistant as well as the control questions on spatial skills and prior knowledge of Grand Theft Auto V were completed by all participants on a computer.

While the present paper focuses on wayfinding performance and usability, the procedure also included additional tasks designed to evaluate internal spatial representations; these data are not presented here.

\subsection{Material}

\subsubsection{Virtual environment conditions based on Grand Theft Auto V}

The two virtual environment conditions are based on two neighboring districts of the fictitious virtual city of Los Santos from the video game Grand Theft Auto V (Rockstar Games, 2013). Grand Theft Auto V is a gaming software that embodies an avatar within a visually realistic environment (e.g., daily solar cycle, pedestrians, vehicles, weather conditions). The experiment was carried out using the "director mode", which 
allows the environmental and visual parameters of Grand Theft Auto $V$ to be controlled. The software was installed on an Asus TUF Gaming FX504 Series PC and displayed on a separate 19-inch screen in front of the participant.

For this study, two virtual neighborhoods that were as similar as possible to the characteristics of European cities were chosen: A high density of landmarks mixing both commercial and residential buildings, including apartments as well as small shops and restaurants, an irregular mapping (urban blocks of different sizes and shapes) and no high-rise buildings. As the names of some shops consisted of French puns, we masked them visually by positioning objects before their facades, in order to avoid any resulting cognitive saliency effect.

For the training stage, we selected two other virtual districts that did not share any landmarks or signs in common with the two experimental districts, in order to avoid any transfer of learning.

The Grand Theft Auto $V$ environment was configured specifically for the experiment so as to be identical across conditions and participants. The settings chosen and corresponding justifications are detailed in Table 1.

\begin{tabular}{|c|c|c|}
\hline Setting & Selected value & Justification \\
\hline Time flow & Frozen & $\begin{array}{l}\text { To keep it constant across all participants and } \\
\text { experimental conditions }\end{array}$ \\
\hline Time schedule & $\begin{array}{l}\text { Eight o'clock in the } \\
\text { morning }\end{array}$ & $\begin{array}{l}\text { To enable the participants to orient themselves in } \\
\text { the environment thanks to the location of the } \\
\text { sun in the east }\end{array}$ \\
\hline Weather & Clear and sunny & $\begin{array}{l}\text { So that the directions of the sun's rays and of } \\
\text { shadows were always visible }\end{array}$ \\
\hline $\begin{array}{l}\text { Pedestrian } \\
\text { density }\end{array}$ & Low & \multirow{2}{*}{$\begin{array}{l}\text { To make the environment vivid with limited risks } \\
\text { of collisions between the participant and non- } \\
\text { player characters }\end{array}$} \\
\hline Traffic density & Low & \\
\hline No go zones & No & \multirow{3}{*}{$\begin{array}{l}\text { To avoid any event involving the avatar and non- } \\
\text { player characters interrupting the experience }\end{array}$} \\
\hline Invincibility & Yes & \\
\hline Wanted level & Deactivated & \\
\hline Point of view & First person & \multirow{3}{*}{ To mimic human vision as closely as possible } \\
\hline Field of view & $\begin{array}{l}85^{\circ} \text { horizontally, } 55^{\circ} \\
\text { vertically }\end{array}$ & \\
\hline Head-up display & Hidden & \\
\hline
\end{tabular}

Table 1: Settings used for the experiment and the values selected for the experiment.

\subsubsection{Navigational assistants}

The two groups of participants used one out of two voice navigational assistants. This protocol, based on the "Wizard of $\mathrm{Oz}$ " technique, gave the navigational assistants the appearance of a fully functional software for the participant, although the features were actually operated discreetly by the experimenter (Salber \& Coutaz, 1993).

A loudspeaker was wired to the experimenter's laptop, in order to broadcast instructions given by the navigational assistant during the wayfinding task. Both assistants used the same synthetic female voice. For both assistants, the formulations and pronunciations (vocabulary, grammar mode, diction) were based on the instructions provided by common navigational aids (GPS, navigation apps). In particular, the instructions given by the survey assistant were all based on the first vocal instruction given for a route by Google Maps, which is the most widely used navigational mobile app according to the tech business magazine The Manifest (Panko, 2018).

\subsubsection{Navigation control}

Navigation within the environment was controlled through the 3D Rudder platform. This is a podal interface through which the participants control the moves of their avatar by rocking and pressing actions 
while seated (Figure 4). This device was chosen to let the participants free of using their hands while navigating. This was due to a condition in the larger study in which the participants had to perform a secondary task with their hands during navigation. This other condition is not analyzed and reported in this paper.

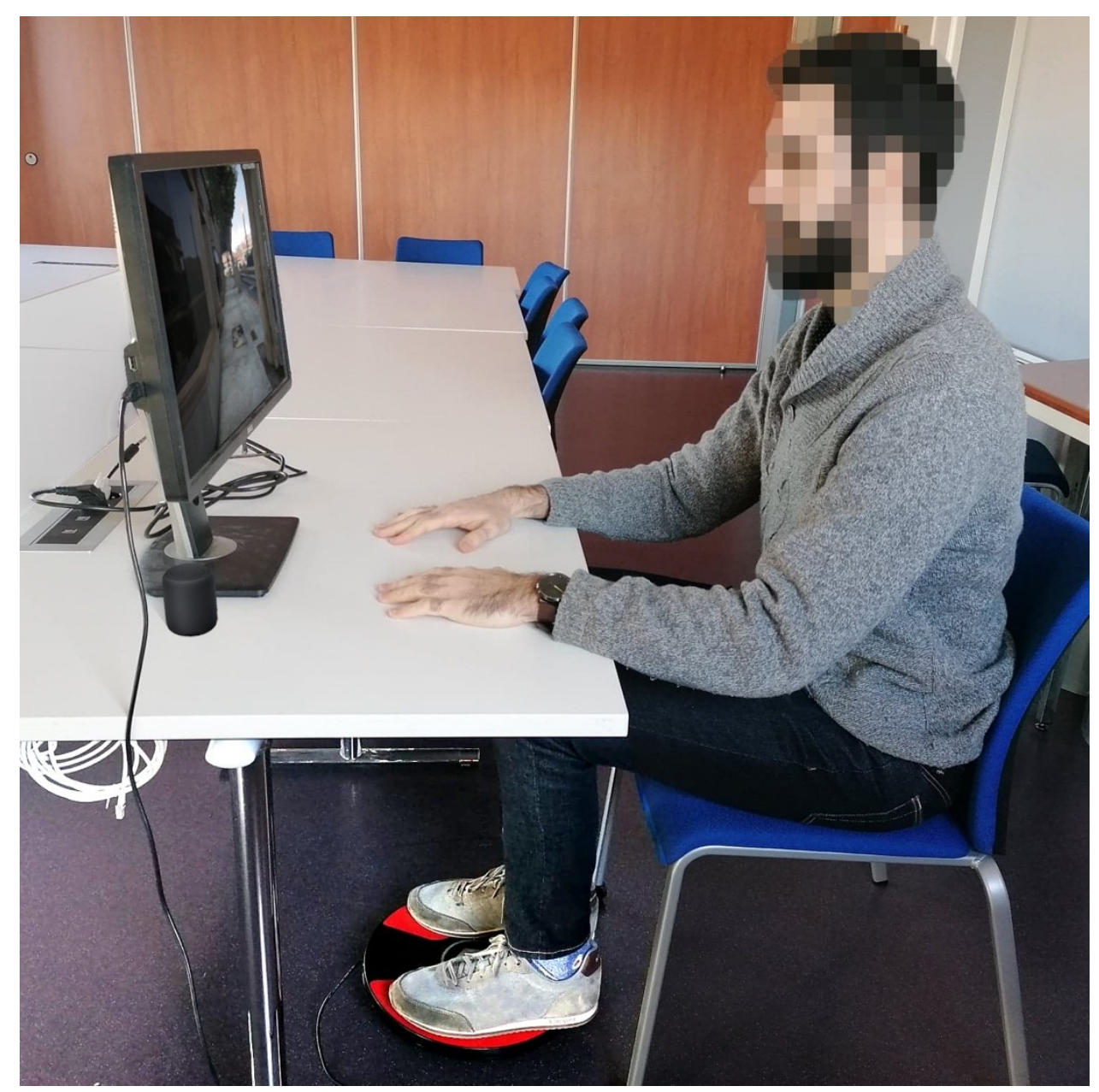

Figure 4: Setup for the navigation control.

The participant is seated and uses the $3 D$ Rudder platform under their feet to move in the virtual environment. The screen in front of the participant displays the environment from their viewpoint. Instructions from the navigational assistant are given to the participant via the loudspeaker on the left.

The speed of the avatar in Grand Theft Auto $V$ was set to a default running pace. Subsequently, the speed was adjusted using the 3D Rudder settings. Each axis of movement (forward - backward, right - left) was limited to $70 \%$ of its maximum value. The walking speed was thus capped at $1.69 \mathrm{~m} / \mathrm{s}$, to be similar to the walking speed of NPCs in the virtual environment. Each axis of rotation of the platform (right - left, up down gaze orientation) was limited to $80 \%$ of its maximum value in order to appear not too slow for the participant, without creating a substantial gap between the rotation speed and the walking speed, to avoid any handling difficulty. In addition, inactive ranges at the beginning of each axis were set in the $3 D$ Rudder settings to avoid triggering accidental movements of the avatar: The two walking axes had an inactive range of $15 \%$, and the two gaze orientation axes had an inactive range of $40 \%$. The sensitivity of all axes was set to the default median value of the $3 D$ Rudder software interface.

\subsubsection{Usability and control questionnaires}

The System Usability Scale (SUS) (Brooke, 1996) is a self-administered questionnaire that assesses, for a given system, the perceived effectiveness, efficiency and user satisfaction with its use. It consists of 10 items in the form of five-point Likert scales and gives a score between 0 and 100 (Figure 5); seven ranks of device usability are distinguished, ranging from the "worst imaginable" (score below 12.5) to the "best 
imaginable" (score above 90.9) (Bangor et al., 2009; Brooke, 2013). Usability is considered "good" from a score of 71.4 .

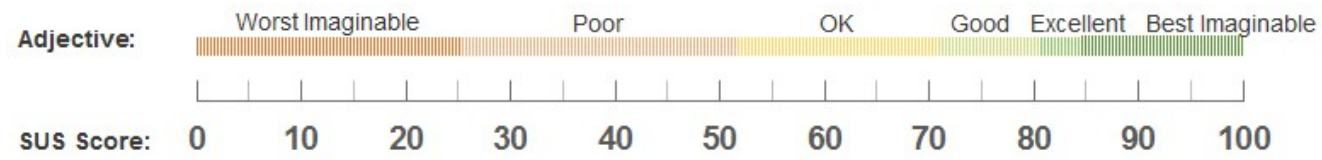

Figure 5: The SUS scale of scores and corresponding ranks of usability (Bangor et al., 2009; Brooke, 2013).

The control questions consisted in an evaluation of the participant's spatial skills and prior use of Grand Theft Auto V. The questionnaire on spatial skills consisted in 16 Likert scales comprising three and five-point items, and measured the use of survey and landmark representations as well as general orientation in everyday life (Pazzaglia et al., 2000). The questions on the participant's prior use of Grand Theft Auto $V$ were designed to assess their degree of mastery of the experimental material before taking the task. The questionnaire comprised a first yes-no question about personal use the participant may have had of Grand Theft Auto $V$ prior to the experiment. If the participant reported having played Grand Theft Auto V, two additional six-point Likert scales measured the participant's perceived knowledge of the game (from "no knowledge" to "perfect knowledge") and the specific prior knowledge about the two experimental neighborhoods (from "I couldn't find my way around at all" to "I found my way around perfectly").

\subsection{Collected data}

During the wayfinding task, the participant's screen and the $3 D$ Rudder status window were simultaneously recorded on video files using Open Broadcaster Software (Figure 6). The purpose of recording the $3 D$ Rudder status window was to allow accurate analysis of the videos by confirming the participant's movement.

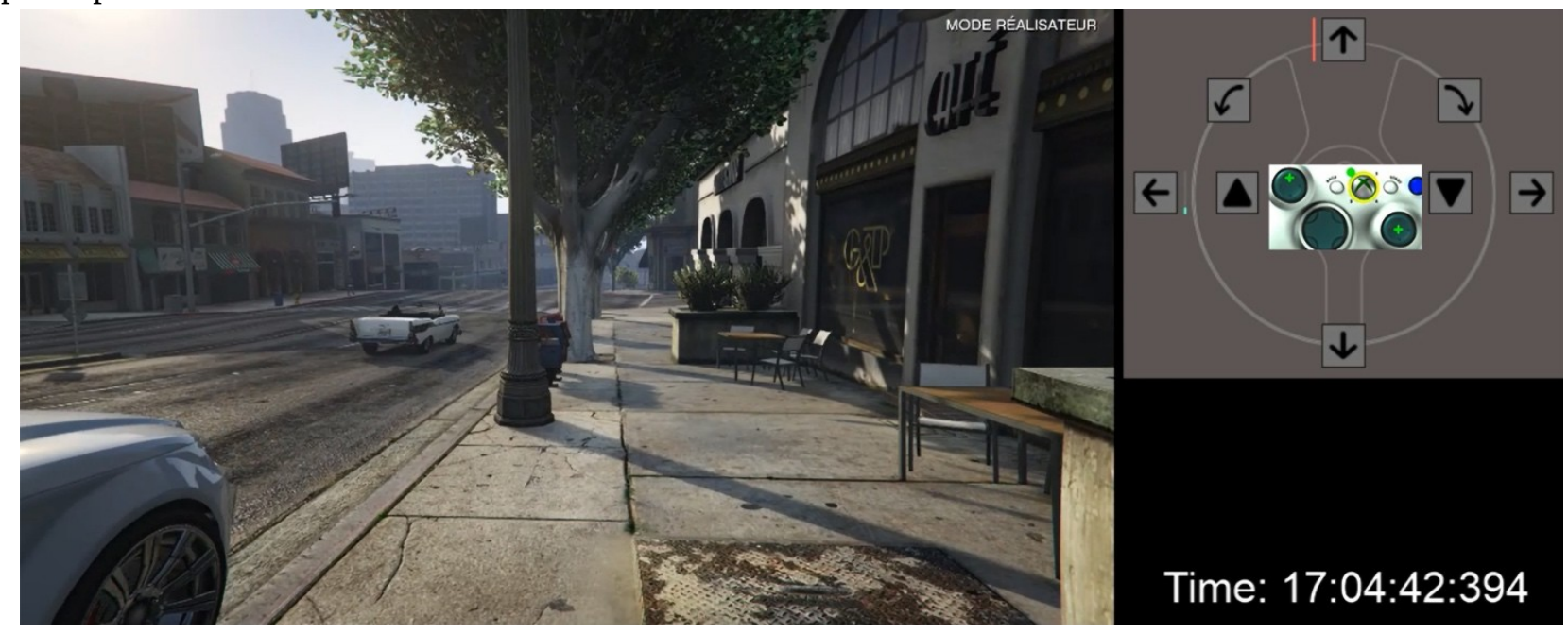

Figure 6: Screen recorded during the experiment.

The left part shows the participant's screen. The other elements are only visible to the experimenter: The upper right part is the $3 D$ Rudder movement display window. At the bottom right, the time is recorded.

Two videos, one for each neighborhood, were recorded for each participant. Ninety-two videos (15 hours) were thus collected for the sample of 46 participants. The questionnaires were completed by all the 46 participants.

\subsection{Analyses}

Wayfinding performance was measured by coding the video recordings, using the Behavioral Observation Research Interactive Software (BORIS) (Friard \& Gamba, 2016). Three concepts were defined for these measurements: "Mistakes", "visual explorations" and "stops". A "mistake" in the wayfinding task 
refers to an event during which the participant moves outside of the path indicated by the navigational assistant, thus making a detour. A "visual exploration" designates a moment during which the participant makes head movements from top to bottom or from right to left. A "stop" refers to a moment during which the participant remains motionless. A stop was recorded as soon as the participant did not push the platform in any direction. The coding was therefore carried out by counting the numbers of mistakes, visual explorations and stops.

In addition, the mean inter-event time intervals between two visual explorations and two stops were measured for each participant. The inter-event time intervals represented the frequency of occurrence of the related behavior. As the total duration of the completion of the trips differed according to the participants, the frequency of occurrence of an event was not related to the number of occurrences of this event. The frequencies of visual explorations and stops were therefore analyzed as separate dependent variables.

Multiple analysis of variances (MANOVA) and analysis of variance (ANOVA) were performed on all data. All distributions met the normality assumption of the analyses, as verified by the Kolmogorov-Smirnov test. The homoscedasticity assumption was not met for three distributions. However, ANOVAs and MANOVAs are robust against violations of homoscedasticity (Allen \& Bennett, 2007; Box, 1953). We therefore chose, as recommended by Box (1953), to perform these robust parametric tests rather than their less robust, non-parametric alternatives.

\section{Results}

\subsection{Wayfinding performance}

A MANOVA on the data collected in both experimental conditions of the wayfinding task was carried out on the following dependent variables: Number of mistakes, number of visual explorations and inter-event time intervals (in seconds) between two visual explorations, number of complete stops and inter-event time intervals (in seconds) between two stops. The analysis indicates a main effect of the navigational assistant conditions (Pillai's trace $=.33, \mathrm{~F}(3.95), p<.01, \eta_{\mathrm{p}}^{2}=.33$ ). Further univariate analyses applying the Benjamini-Hochberg multiple comparison correction with a false discovery rate of .05 indicate an effect across all variables (Benjamini \& Hochberg, 1995).

Participants made fewer mistakes with the landmark assistant than with the survey assistant.

Participants also made fewer visual explorations with the landmark assistant than with the survey assistant, and less frequently with the landmark assistant than with the survey assistant.

Finally, participants made fewer stops with the landmark assistant than with the survey assistant, and less frequently with the landmark assistant than with the survey assistant. These results are detailed in Table 2.

\begin{tabular}{|c|c|c|c|c|c|}
\hline Variable & $\begin{array}{l}\text { Landmar } \\
\text { k } \\
\text { assistant }\end{array}$ & $\begin{array}{l}\text { Survey } \\
\text { assistant }\end{array}$ & $\begin{array}{l}\text { df } \\
\text { (Residua } \\
\text { Is) }\end{array}$ & $\mathbf{F}$ & $\begin{array}{l}\text { Significance } \\
\text { and effect } \\
\text { size }\end{array}$ \\
\hline Mistakes & $\begin{array}{l}M=3.96 \\
S D=3.85\end{array}$ & $\begin{array}{l}M=8.41 \\
S D=7.63\end{array}$ & $\begin{array}{l}1 \\
(44)\end{array}$ & 6.40 & $\begin{array}{l}* \\
\eta^{2}=.13\end{array}$ \\
\hline Visual explorations & $\begin{array}{l}M=25.5 \\
S D=8.5\end{array}$ & $\begin{array}{l}M=36.0 \\
S D=10.3\end{array}$ & $\begin{array}{l}1 \\
(44)\end{array}$ & 14.60 & $\begin{array}{l}* * * \\
\eta^{2}=.25\end{array}$ \\
\hline $\begin{array}{l}\text { Visual explorations } \\
\text { inter-event time } \\
\text { interval }\end{array}$ & $\begin{array}{l}M=38.8 \\
S \\
S D=11.3 \\
S\end{array}$ & $\begin{array}{l}M=30.6 \mathrm{~s} \\
S D=6.3 \mathrm{~s}\end{array}$ & $\begin{array}{l}1 \\
(44)\end{array}$ & 8.91 & $\begin{array}{l}* * \\
\eta^{2}=.17\end{array}$ \\
\hline Stops & $\begin{array}{l}M=16.2 \\
S D=5.93\end{array}$ & $\begin{array}{l}M=25.5 \\
S D=10.7\end{array}$ & $\begin{array}{l}1 \\
(44)\end{array}$ & 13.64 & $\begin{array}{l}* * * \\
\eta^{2}=.24\end{array}$ \\
\hline $\begin{array}{l}\text { Stops inter-event time } \\
\text { interval }\end{array}$ & $\begin{array}{l}M=68.4 \\
S \\
S D=18.6 \\
S\end{array}$ & $\begin{array}{l}M=54.5 \mathrm{~s} \\
S D=13.4 \mathrm{~s}\end{array}$ & $\begin{array}{l}1 \\
(44)\end{array}$ & 8.26 & $\begin{array}{l}* * \\
\eta^{2}=.16\end{array}$ \\
\hline
\end{tabular}


Table 2: Means and standard deviations of the wayfinding performance variables collected in the landmark assistant condition and the survey assistant condition together with the results of the MANOVA.

Statistically significant p-values are flagged $\left(* p<.05,{ }^{* *} p<.01,{ }^{* * *} p<.001\right)$.

\subsection{User experience: Usability of the navigational assistants}

An ANOVA on the SUS scores in both experimental conditions indicated a main effect of the navigational assistant $\left(\mathrm{F}(1,44)=10.2, p<.01, \eta^{2}=.19\right)$. Participants assessed the usability of the landmark assistant as better than that of the survey assistant. The results are detailed in Table 3.

\begin{tabular}{llllll} 
Variable & $\begin{array}{l}\text { Landmark } \\
\text { assistant }\end{array}$ & $\begin{array}{l}\text { Survey } \\
\text { assistan } \\
\text { t }\end{array}$ & $\begin{array}{l}\text { df } \\
\text { (Residua } \\
\text { Is) }\end{array}$ & F & $\begin{array}{l}\text { Significance } \\
\text { and effect size }\end{array}$ \\
\hline \multirow{2}{*}{ SUS score } & $M=75.5$ & $M=57.6$ & 1 & & $* *$ \\
& SD $=16.0$ & SD $=$ & $(44)$ & 10.2 & $\eta^{2}=.19$
\end{tabular}

Table 3: Results of the univariate analyses of the ANOVA on the SUS scores.

Statistically significant $\mathrm{p}$-values are flagged $\left(* p<.05,{ }^{* *} p<.01\right.$, *** $\left.p<.001\right)$.

\subsection{Control questions: Prior knowledge of Grand Theft Auto $\boldsymbol{V}$ and spatial skills evaluation}

An ANOVA on the answers to the control questions about personal use and prior knowledge of Grand Theft Auto V (map and general knowledge about the game) did not indicate a difference between the groups. An additional correlational analysis between the answers to these questions and observed behaviors during the tasks indicated no correlation between prior knowledge and use of the game and performance on all variables (mistakes, stops and visual explorations). An ANOVA on the scores obtained in the questionnaire on spatial skills did not indicate a difference between the groups.

\section{Discussion}

This study aimed primarily to test the induced performance and perceived usability of a landmarkbased wayfinding aid. By doing so, we also wanted to operate and subsequently assess a video game-based methodology that could represent a starting point for studies in spatial navigation. We used performance measurements on video data, as well as a usability questionnaire, to identify how navigational aids shaped spatial navigation in the virtual environment of Grand Theft Auto $V$ and what type of navigational assistant would be judged the most usable. Our analyses on the data collected highlight the benefits of the landmarkbased aid in wayfinding. As these results provide converging evidence with the literature on wayfinding, they also emphasize the satisfactory methodological qualities of a video game-based methodology.

\subsection{Wayfinding performance: Widespread positive effect of the landmark assistant on effectiveness and efficiency of the trip}

Two navigational assistants were compared in this study. One represented an aid whose principles were based on the literature on the use of landmarks in wayfinding. The second was a survey-based aid similar to the most commonly-used aids available to the general public. Based on video data of the trips completed by the participants, we conducted analyses on three observable behaviors: Making a mistake, visually exploring the environment and stopping completely. For these three observable behaviors, several differences were observed between participants using these two navigational assistants.

Firstly, regarding the effectiveness of the trip, participants using the landmark assistant made fewer mistakes than participants using the survey assistant. This result provides further evidence that the provision of landmark information can ease wayfinding (Denis, 1997; Denis et al., 2007, 2014). Our result therefore suggests that when aiming at increasing the effectiveness of wayfinding by decreasing the number of mistakes an individual can make while following directions, a navigational aid should consider landmarkbased directions.

A second point deals with the efficiency of the trip. While making a mistake and taking the wrong direction are an obstacle to reaching a destination, the notion of wayfinding relies heavily on decision- 
making and orientation, which take place throughout the journey (Montello, 2017; Vandenberg, 2016). A wayfinding difficulty can therefore arise not only as a mistake, but also as a lack of efficiency in the completion of the journey. As it turns out, participants who used the survey assistant had to make more visual explorations of their surroundings, as well as more stops, than participants using the landmark assistant. They also had to perform stops and visual explorations more frequently, resulting in a more hesitant and intermittent trip. These hesitations could be explained by a difficulty in decision making and orientation (Vandenberg, 2016). Our results therefore suggest that this landmark-based wayfinding aid favors these key wayfinding components, as participants need to stop or to look around less while attempting to reach their destination.

Our results substantiate the literature on wayfinding, which emphasizes the use of landmark-based representations as the most cognitively useful for wayfinding purposes. Importantly, as the survey assistant was based on actual instructions given by the most commonly-used navigational aids, our results provide empirical evidence that aids based on survey representations are not optimal and that users of such aids can be outperformed by users of a landmark-based navigational aid when it comes to life-like wayfinding situations.

\subsection{Usability: A clear preference for the landmark assistant}

While objectively-measured effectiveness and efficiency on a given task indeed play a part in the experience a user will have of any device, subjective evaluations of these parameters as well as satisfaction measure the actual usability of such device (Brooke, 2013). As Brooke (2013) stated, there is indeed a possibility that a system allows its users to effectively complete their tasks, but at the expense of much time and effort that lead to it being unused. The usability of both navigational assistants was therefore assessed in order to deepen the analysis of their effect on overall user experience. Our results indicate that participants from both groups evaluated the usability of the navigational assistant they were given differently: The landmark assistant was judged as "good" in average, highlighting a high acceptability of this aid, while the survey assistant was evaluated only as "OK”, suggesting low acceptability.

These results complete those on performance, as they show that not only does a landmark-based aid objectively increase wayfinding effectiveness and efficiency, but also that it is subjectively evaluated as more efficient, more effective and more satisfactory than a survey-based aid. A possible explanation for this satisfaction with the landmark assistant could lie in the fact that this aid closely resembles the way actual people give directions in real life (Denis et al., 2007), therefore making the interaction with the device more natural.

\subsection{Interest and methodological qualities of a video game-based methodology}

To our knowledge, this study is one of the first that makes use of a commercially available video game to implement life-like wayfinding tasks. Our results provide evidence on several methodological interests of the use of a video game as a research tool, including validity, fidelity, transparency, replicability and controllability.

Firstly, the virtual environment of the video game achieves experimental validity. The positive effects of a landmark-based navigational assistant on wayfinding performance, expected to occur in real-world conditions (Denis et al., 2007), indeed take place in the game. This result therefore suggests the psychological fidelity of the video game, as it triggers life-like behavioral responses (Leplat, 1997). This psychological fidelity is favored thanks to the fact that Grand Theft Auto $V$ comes with a high degree of physical and spatial fidelity to the real world. Further studies should investigate this aspect in greater depth by comparing task performance in real and video game environments, in order to assess not only the detection of an effect in the two settings, but also to compare the associated effect sizes in the two conditions.

Moreover, the virtual environment provided by the video game enabled us to provide a transparent description of its use, allowing for replication of the task in the same settings. Unlike studies on spatial navigation that used modified video game frameworks to build new environments (e.g. Ledoux et al., 2014), 
the present study uses the actual virtual environment of Grand Theft Auto V. Only one environmental element was modified in-game, as the names of a few shops consisting in French puns were masked by placing objects before them in order to avoid a cognitive saliency effect. The exact virtual environment used in this study can therefore be observed and walked through by any user of Grand Theft Auto V.

Finally, the controllability of the video game allowed us to operate the wayfinding task by setting all the useful environmental parameters. Thus, elements relevant to the task itself, whether related to the environment (e.g. time of day, weather) or to the visual display (e.g. point of view, head-up display) were adaptable from the game interface. It is worth highlighting that apart from the modification of the walking speed carried out from the $3 D$ Rudder platform, all the desired settings were implemented in this study thanks to the actual features provided by the video game. No additional laboratory-made customization had to be implemented, thus substantially reducing the burden usually associated with the development of virtual environments.

\subsection{Conclusion}

This study aimed at assessing the usability and performance induced by a landmark-based navigational aid. By doing so in the virtual environment of a widely available video game, Grand Theft Auto V, we wanted to explore the interest and the feasibility of a video-game based methodology for a study on spatial navigation. Our results provide converging evidence with the literature that a landmark-based aid allows for better wayfinding performance as well as better usability than survey-based aids. Our video game-based methodology allowed us to put forward several arguments towards the use of such experimental tools for future research.

Some limitations should however be noted. While the survey assistant was based on the most commonly-found navigational aids, it relied only on vocal features in this study. Most current navigational aids indeed offer vocal features that closely resemble the instructions we used in this study; however, they often provide an additional bird's-eye view map that can be looked at simultaneously. Yet, while the simultaneous use of these two types of information can indeed ease the understanding of the given directions, they both consist in survey representations. Our study aimed at evaluating the perceived usability and performance induced by a prototypal aid based on landmark representations, therefore using a simpler, vocalonly assistant. Further studies should therefore explore the usability of such a landmark-based aid with a visual interface incorporating landmarks, in order to compare this solution with actual existing aids. Furthermore, while Grand Theft Auto V provides a true-to-life virtual environment, its virtual city of Los Santos is based on North American urban environments. While we used two neighborhoods resembling European urban settings, some differences might exist between these areas and the actual French cities our participants walk through daily. Further studies should pursue this question further in order to confirm the usability of a video game-based methodology for international audiences.

Considering its qualities, this video game-based approach nevertheless represents a low-cost turnkey solution to studies on spatial navigation, overcoming common barriers associated with the development of large and vivid environments, as well as possessing substantial methodological qualities.

\section{License agreement statement}

Take-Two Interactive Software, Inc. is the worldwide owner of all intellectual property rights in the Grand Theft Auto video game series, including without limitation all copyrights, trademarks, and trade dress associated with the video game. The use of such intellectual property in connection with this study has been licensed by Take-Two Interactive Software, Inc.

\section{Acknowledgments}

The authors would like to thank Take-Two Interactive, Inc. for allowing us to use Grand Theft Auto V for this study. The authors express their especial thanks to Mrs. Gena Feist, from the New York City office, for taking the time to draft, review and validate all the legal documents. 
The authors would also like to express their sincere thanks to the team at 3D Rudder, and especially Mr. Emmanuel Fromy, for their significant help regarding the 3D Rudder platform setup for this study.

\section{References}

Allen P.J. and Bennett K., 2007. SPSS for the Health and Behavioural Sciences. Thomson Learning.

Bangor A., Kortum, P., Miller J., 2009. Determining What Individual SUS Scores Mean: Adding an Adjective Rating Scale. Journal of Usability Studies, 4(3), 10.

Benjamini Y. and Hochberg Y., 1995. Controlling the False Discovery Rate: A Practical and Powerful Approach to Multiple Testing. Journal of the Royal Statistical Society: Series B (Methodological), 57(1), 289-300. doi:10.1111/j.2517-6161.1995.tb02031.x

Bohil C.J., Alicea B., Biocca F.A., 2011. Virtual reality in neuroscience research and therapy. Nature Reviews Neuroscience, 12(12), 752-762. doi:10.1038/nrn3122

Box G.E.P., 1953. Non-Normality and Tests on Variances. Biometrika, 40(3/4), 318-335. JSTOR. doi:10.2307/2333350

Brooke J., 1996. SUS-A quick and dirty usability scale. In Usability evaluation in industry (CRC Press, p. 189-194).

Brooke J., 2013. SUS : A Retrospective. J. Usability Studies, 8(2), 29-40.

Burkhardt, J.-M. (2003). Réalité virtuelle et ergonomie: Quelques apports réciproques. Le travail humain, Vol. 66(1), 65-91.

Coutrot A., Schmidt S., Coutrot L., Pittman J., Hong L., Wiener J.M., Hölscher C., Dalton R.C., Hornberger M., Spiers H.J., 2019. Virtual navigation tested on a mobile app is predictive of real-world wayfinding navigation performance. PLOS ONE, 14(3), e0213272. doi:10.1371/journal.pone.0213272

Denis M., 1997. The description of routes: A cognitive approach to the production of spatial discourse. Cahiers de psychologie cognitive, 16(4), 409-458.

Denis M., Michon P.-E., Tom A., 2007. Assisting pedestrian wayfinding in urban settings : Why references to landmarks are crucial in direction-giving. Applied spatial cognition: From research to cognitive technology, 25-51.

Denis M., Mores C., Gras D., Gyselinck V., Daniel M.-P., 2014. Is Memory for Routes Enhanced by an Environment's Richness in Visual Landmarks? Spatial Cognition \& Computation, 14(4), 284-305. doi:10.1080/13875868.2014.945586

Friard O. and Gamba M., 2016. BORIS: a free, versatile open-source event-logging software for video/audio coding and live observations. Methods in Ecology and Evolution, 7(11), 1325-1330. doi:10.1111/2041-210X.12584

Gibson J.J., 1979. The Ecological Approach to Visual Perception: Classic Edition. Psychology Press. doi:10.4324/9781315740218

Golledge R.G., 1992. Place recognition and wayfinding : Making sense of space. Geoforum, 23(2), 199-214. doi:10.1016/0016-7185(92)90017-X

Grison E. and Gyselinck V., 2019. La cognition spatiale pour repenser les aides à la navigation. LAnnee psychologique, Vol. 119(2), 243-278.

Gyselinck V., Meneghetti C., Bormetti M., Orriols E., Piolino P., De Beni R., 2013. Considering spatial ability in virtual route learning in early aging. Cognitive Processing, 14(3), 309-316. doi:10.1007/s10339-013-0557-1

Johnson-Laird P.N., 1980. Mental models in cognitive science. Cognitive Science, 4(1), 71-115. doi:10.1016/S0364-0213(81)80005-5

Khan N. and Rahman A.U., 2018. Rethinking the Mini-Map: A Navigational Aid to Support Spatial Learning in Urban Game Environments. International Journal of Human-Computer Interaction, 34(12), 1135-1147. doi:10.1080/10447318.2017.1418804 
Kimura K., Reichert J.F., Olson A., Pouya O.R., Wang X., Moussavi Z., Kelly D.M., 2017. Orientation in Virtual Reality Does Not Fully Measure Up to the Real-World. Scientific Reports, 7(1), 18109. doi:10.1038/s41598-017-18289-8

Ledoux A.-A., Boyer P., Phillips J.L., Smith A., Labelle A., Bohbot V.D., 2014. Structural Hippocampal Anomalies in a Schizophrenia Population Correlate with Navigation Performance on a Wayfinding Task. Frontiers in Behavioral Neuroscience, 8. doi:10.3389/fnbeh.2014.00088

Leplat J., 1997. Simulation et simulateur: Principes et usages. In Regards sur l'activité en situation de travail : Contribution à la psychologie ergonomique (p. 157-181). PUF.

Lhuillier S., Gyselinck V., Dutriaux L., Grison E., Nicolas S., 2018. "Like a ball and chain” : Altering locomotion effort perception distorts spatial representations. Journal of Environmental Psychology, 60, 63-71. doi:10.1016/j.jenvp.2018.10.008

Li H., Thrash T., Hölscher C., Schinazi V.R., 2019. The effect of crowdedness on human wayfinding and locomotion in a multi-level virtual shopping mall. Journal of Environmental Psychology, 65, 101320. doi:10.1016/j.jenvp.2019.101320

Lingwood J., Blades M., Farran E.K., Courbois Y., Matthews D., 2018. Using virtual environments to investigate wayfinding in 8- to 12-year-olds and adults. Journal of Experimental Child Psychology, 166, 178-189. doi:10.1016/j.jecp.2017.08.012

Meneghetti C., Labate E., Pazzaglia F., Hamilton C., Gyselinck V., 2017. The role of visual and spatial working memory in forming mental models derived from survey and route descriptions. British Journal of Psychology, 108(2), 225-243. doi:10.1111/bjop.12193

Montello D.R., 2005. Navigation. In The Cambridge Handbook of Visuospatial Thinking (p. 257-294). Cambridge University Press. doi:10.1017/CBO9780511610448.008

Montello D.R., 2017. Cognition and Spatial Behavior. In International Encyclopedia of Geography (p. 1-20). American Cancer Society. doi:10.1002/9781118786352.wbieg0498

Nys M., Hickmann M., Gyselinc V., 2018. The role of verbal and visuo-spatial working memory in the encoding of virtual routes by children and adults. Journal of Cognitive Psychology, 30(7), 710-727. doi:10.1080/20445911.2018.1523175

Panko R., 2018. The Popularity of Google Maps: Trends in Navigation Apps in 2018. The Manifest. https://themanifest.com/app-development/popularity-google-maps-trends-navigation-apps-2018

Pazzaglia F., Cornoldi C., Beni R.D., 2000. Differenze individuali nella rappresentazione dello spazio e nell'abilità di orientamento : Presentazione di un questionario autovalutativo. Giornale italiano di psicologia, 3/2000. doi:10.1421/310

Pazzaglia F., Meneghetti C., Labate E., Ronconi L., 2017. Are Wayfinding Self-efficacy and Pleasure in Exploring Related to Shortcut Finding? A Study in a Virtual Environment. In T. Barkowsky, H. Burte, C. Hölscher, \& H. Schultheis (Éd.), Spatial Cognition X (p. 55-68). Springer International Publishing. doi:10.1007/978-3-319-68189-4_4

Plancher G., Gyselinck V., Piolino P., 2018. The Integration of Realistic Episodic Memories Relies on Different Working Memory Processes : Evidence from Virtual Navigation. Frontiers in Psychology, 9. doi:10.3389/fpsyg.2018.00047

Salber D. and Coutaz J., 1993. Applying the Wizard of Oz technique to the study of multimodal systems. In L. J. Bass, J. Gornostaev, \& C. Unger (Éd.), Human-Computer Interaction (Vol. 753, p. 219-230). Springer Berlin Heidelberg. doi:10.1007/3-540-57433-6_51

Sharma G., Kaushal Y., Chandra S., Singh V., Mittal A.P., Dutt V., 2017. Influence of Landmarks on Wayfinding and Brain Connectivity in Immersive Virtual Reality Environment. Frontiers in Psychology, 8. doi:10.3389/fpsyg.2017.01220

Siegel A.W. and White S.H., 1975. The Development of Spatial Representations of Large-Scale Environments. In H. W. Reese (Éd.), Advances in Child Development and Behavior (Vol. 10, p. 9-55). JAI. doi:10.1016/S0065-2407(08)60007-5

Tarr M.J. and Warren W.H., 2002. Virtual reality in behavioral neuroscience and beyond. Nature Neuroscience, 5(S11), 1089-1092. doi:10.1038/nn948 
Tolman E.C., 1948. Cognitive maps in rats and men. Psychological Review, 55(4), 189-208. doi:10.1037/h0061626

Vandenberg A.E., 2016. Human Wayfinding: Integration of Mind and Body. In R. H. Hunter, L. A. Anderson, \& B. L. Belza (Éd.), Community Wayfinding: Pathways to Understanding (p. 17-32). Springer International Publishing. doi:10.1007/978-3-319-31072-5_2

Wiener J.M., Büchner S.J., Hölscher C., 2009. Taxonomy of Human Wayfinding Tasks : A Knowledge-Based Approach. Spatial Cognition \& Computation, 9(2), 152-165. doi:10.1080/13875860902906496

Wilson P.N., 2013. Use of Virtual Reality Computing in Spatial Learning Research. Handbook Of Spatial Research Paradigms And Methodologies. doi:10.4324/9780203727089-12 